\section{Omalizumab as a Provoking Factor for Venous Thromboembolism}

\author{
Crhistian-Mario Oblitas ${ }^{1}$, Francisco Galeano-Valle ${ }^{1,2}$ (i), \\ Laura Vela-De La Cruz'1, Jorge Del Toro-Cervera ${ }^{1,2}$ \\ and Pablo Demelo-Rodríguez ${ }^{1,2}$
}

`Venous Thromboembolism Unit, Hospital General Universitario Gregorio Marañón, Madrid, Spain. ${ }^{2}$ Instituto de Investigación Sanitaria Gregorio Marañón (IiSGM), Madrid, Spain.
Drug Target Insights

Volume 13: 1-2

(C) The Author(s) 2019

Article reuse guidelines:

sagepub.com/journals-permissions

DOI: $10.1177 / 1177392819861987$

(S)AGE

\begin{abstract}
A 43-year-old man with a history of severe extrinsic allergic asthma treated with once-monthly omalizumab (600 mg) for the last 15 months. He presented to the emergency room with a 2-week history of right lower limb pain and chest pleuritic pain. Computed tomography pulmonary angiography showed bilateral pulmonary embolism with right-sided pulmonary infarction and ultrasound of right lower limb confirmed distal deep vein thrombosis. No other known risk factors were identified. Treatment with omalizumab was stopped during hospitalization. The Naranjo Adverse Drug Reaction (ADR) Probability Scale classifies this as a probable ADR (score of 6). Omalizumab is a humanized monoclonal anti-IgE antibody indicated for the treatment of persistent moderate-to-severe asthma and certain chronic refractory urticaria. The EXCELS study (The Epidemiologic Study of Xolair (omalizumab): Evaluating Clinical Effectiveness and Long-term Safety in Patients with Moderate-to-Severe Asthma), a postmarketing observational cohort study to assess clinical safety profile of omalizumab, showed a significant increase in venous thromboembolism. In conclusion, omalizumab has been associated with arterial and venous thromboembolic events, although the evidence is not definitive.
\end{abstract}

KEYWORDS: omalizumab, venous thromboembolism

RECEIVED: May 31, 2019. ACCEPTED: June 11, 2019.

TYPE: Letter to the Editor

FUNDING: The author(s) received no financial support for the research, authorship, and/or publication of this article.
DECLARATION OF CONFLICTING INTERESTS: The author(s) declared no potential conflicts of interest with respect to the research, authorship, and/or publication of this article.

CORRESPONDING AUTHOR: Pablo Demelo-Rodríguez, Venous Thromboembolism Unit, Hospital General Universitario Gregorio Marañón, C/. Doctor Esquerdo, 46, 28007 Madrid, Spain. Email: pbdemelo@hotmail.com
Venous thromboembolism (VTE), that includes deep vein thrombosis (DVT) and pulmonary embolism (PE), is a common and potentially fatal disease. The cause of VTE includes cancer, systemic, surgical, and medication-related conditions and its treatment is based on anticoagulation. ${ }^{1}$

We present the case of a 43-year-old man with a history of severe extrinsic allergic asthma treated with once-monthly omalizumab $(600 \mathrm{mg})$ for the last 15 months. He was not receiving other treatments. He presented to the emergency room with a 2-week history of right lower limb pain and chest pleuritic pain. On admission, blood pressure was 160/94 mm $\mathrm{Hg}$, heart rate was $93 \mathrm{bpm}$, and basal oxygen saturation was 98\%. The remainder of physical examination was normal and no signs of DVT were found. C-reactive protein was $3.2 \mathrm{mg} / \mathrm{dL}$ (normal $<0.5 \mathrm{mg} / \mathrm{dL}$ ) and D-dimer was reported as $5.113 \mathrm{ng} /$ $\mathrm{mL}$ (normal < 250). The CT pulmonary angiography showed bilateral $\mathrm{PE}$ with right-sided pulmonary infarction and ultrasound of right lower limb confirmed distal DVT. He was admitted and low molecular weight heparin was started. Pulmonary Embolism Severity Index (PESI) score was 53 points (class I mortality risk). Echocardiogram showed mild dilation of the right ventricle, the right-to-left ventricle diameter ratio was 0.85 (normal), with normal systolic function and normal tricuspid annular plane systolic excursion $(22 \mathrm{~mm})$. The patient's hospital stay was uneventful and he was discharged 4 days later under treatment with rivaroxaban. Treatment with omalizumab was stopped. No known risk factors were identified: his body mass index was normal $\left(24 \mathrm{~kg} / \mathrm{m}^{2}\right)$ and he denied any history of smoking; he had not recent history of immobilization or prolonged travel; no family history of VTE was present. A colonoscopy was normal, and prostate-specific antigen was within normal range $(0.41 \mu \mathrm{g} / \mathrm{L})$. Thrombophilia testing including antithrombin, homocysteine, protein $\mathrm{C}$ and $\mathrm{S}$, antiphospholipid antibodies, factor $\mathrm{V}$ Leiden, and prothrombin mutation was performed 3 months after the VTE event, and it was normal. Anticoagulation was maintained for 6 months. C-reactive protein was $0.3 \mathrm{mg} / \mathrm{dL}$ and D-dimer was $90 \mathrm{ng} / \mathrm{mL}$ after 3 months of the VTE episode. The Naranjo Adverse Drug Reaction (ADR) Probability Scale classifies this as a probable ADR (score of 6: there are previous conclusive reports on this reaction $(+1)$, the adverse even appeared after the suspected drug was administered $(+2)$, it improved after the drug was discontinued $(+1)$, there were not alternative causes $(+2)) .^{2}$

Omalizumab is a humanized monoclonal anti- $\mathrm{IgE}$ antibody indicated for the treatment of persistent moderate-to-severe asthma and certain chronic refractory urticaria. It has shown reduction in severity, exacerbation of symptoms, and the use of corticosteroids and improvement in quality of life. Omalizumab plays a major role in chronic allergic inflammatory processes acting both innate and humoral immunity. Studies have shown a link between D-dimer levels and symptomatic load in chronic urticaria, and both features improve in patients taking 
omalizumab. ${ }^{3,4}$ The efficacy of omalizumab in these disorders has been evaluated by observing symptomatic improvement and decreased levels of D-dimer, by mechanisms not well known. It has been suggested that this effects could simply reflect the reduction of the general inflammatory state of the disease. In this context, active chronic urticaria and persistent allergic asthma are associated with a procoagulant state by activating the extrinsic pathway of coagulation in relation to high levels of circulatory $\mathrm{IgE}$, molecule which is blocked by Omalizumab reducing levels of D-dimer. Paradoxically, it does not result in decreasing the risk of VTE. ${ }^{4,5}$ The D-dimer levels are considered to be useful for the diagnosis of thrombosis, and they can be clinically used due to its high negative predictive value. Hence, the decrease in $\mathrm{D}$-dimer levels could mask its diagnostic predictive value in cases of VTE in patients receiving omalizumab.

Recently, some articles reporting the association of omalizumab with pulmonary vein thrombosis ${ }^{6}$ and some studies evaluating the long-term use of omalizumab have been published.The most important study to date is the EXCELS study, a postmarketing 5-year follow-up observational cohort study to assess clinical safety profile of omalizumab (7857 patients were included). This study, primarily designed for the detection of malignancies, showed an increased risk in VTE (crude incidence rate $=3.2[95 \% \mathrm{CI}=2.4-4.3]$ vs $1.5[95 \% \mathrm{CI}=0.8$ 2.5] per 1000 person-years). The incidence rate of primary neoplasms was similar in both groups. ${ }^{7}$ Consequently, the US Food and Drug Administration ${ }^{8}$ published a Drug Safety Communication on September 26, 2014, describing slightly higher risk of blood clots in the lungs and veins, in addition to heart and brain adverse events. However, conflicting results of other recent studies have been published. A letter reported results from a pooled analysis of cardiovascular events from 25 randomized controlled trials and 2 extension studies of omalizumab. This analysis did not find any differences in the incidence of VTE between omalizumab or placebo. ${ }^{9}$

There are no specific recommendations for patients who suffer a VTE event under treatment with omalizumab, but if we consider it as a temporary risk factor for VTE, we suggest drug withdrawal and anticoagulation for at least 6 months in the absence of other risk factors. ${ }^{10}$

Because omalizumab is administered in a health care setting, it may not be listed on the patient's home medication list, and therefore, physicians and pharmacists should evaluate clinicadministered medication when evaluating VTE risk factors.
In conclusion, omalizumab has been associated with arterial and venous thromboembolic events, although the evidence is not definitive. It has been associated with decrease in D-dimer levels that could mask its diagnostic predictive value. We point out the need for studies that clarify the role of omalizumab in the setting of VTE.

\section{Author Contributions}

All authors contributed equally to the colletion of data, analysis and interpretation, manuscript writing and final approval of manuscript.

\section{Informed Consent}

We obtained the patient's written informed consent before the submission of the letter.

\section{ORCID iD}

Francisco Galeano-Valle (D) https://orcid.org/0000-0003-1321 $-6866$

\section{REFERENCES}

1. Tritschler T, Kraaijpoel N, Le Gal G, Wells PS. Venous thromboembolism: advances in diagnosis and treatment. JAMA. 2018;320:1583-1594.

2. Naranjo CA, Busto U, Sellers EM, Sandor P, Ruiz I, Roberts EA. A method for estimating the probability of adverse drug reactions. Clin Pharmacol Ther. 1981;30:239-245.

3. D'Amato G, Stanziola A, Sanduzzi A, et al. Treating severe allergic asthma with anti-IgE monoclonal antibody (omalizumab): a review. Multidiscip Respir Med. 2014;9:23.

4. Asero R. Serial D-dimer plasma levels in a patient with chronic spontaneous urticaria developing resistance to omalizumab. Clin Exp Dermatol. 2017;42:667-669.

5. Yalcin AD, Celik B, Gumuslu S. D-dimer levels decreased in severe allergic asthma and chronic urticaria patients with the omalizumab treatment. Expert Opin Biol Ther. 2014;14:283-286.

6. Narukonda S, Vinod NR, Joshi M. A case of pulmonary vein thrombosis associated with treatment of omalizumab. J Investig Med High Impact Case Rep. 2017;5:2324709617724176.

7. Iribarren C, Rahmaoui A, Long AA, et al. Cardiovascular and cerebrovascular events among patients receiving omalizumab: results from EXCELS, a prospective cohort study in moderate to severe asthma. J Allergy Clin Immunol. 2017;139:1489.e5-1495.e5.

8. US Food and Drug Administration. FDA drug safety communication: FDA approves label changes for asthma drug Xolair (omalizumab), including describing slightly higher risk of heart and brain adverse events. Website. https://www.fda .gov/Drugs/DrugSafety/ucm414911.htm. Updated September, 2014. Accessed January 22, 2019.

9. Iribarren C, Rothman KJ, Bradley MS, Carrigan G, Eisner MD, Chen H. Cardiovascular and cerebrovascular events among patients receiving omalizumab: pooled analysis of patient-level data from 25 randomized, double-blind, placebocontrolled clinical trials. J Allergy Clin Immunol. 2017;139:1678-1680.

10. Peñaloza-Martínez E, Demelo-Rodríguez P, Proietti $M$, et al. Update on extended treatment for venous thromboembolism. Ann Med. 2018;50:666-674. 